Research Article

\title{
New Contour Design Method for Rocket Nozzle of Large Area Ratio
}

\author{
Dechuan Sun $\mathbb{D}^{1,2}$ Tianyou Luo, ${ }^{1}$ and Qiang Feng ${ }^{1}$ \\ ${ }^{1}$ School of Aeronautics and Astronautics, Dalian University of Technology, Dalian 116024, China \\ ${ }^{2}$ Key Laboratory of Advanced Technology for Aerospace Vehicles, Liaoning Province 116024, China \\ Correspondence should be addressed to Dechuan Sun; dechuans@dlut.edu.cn
}

Received 3 September 2019; Revised 13 November 2019; Accepted 2 December 2019; Published 20 December 2019

Academic Editor: Wen Bao

Copyright (C) 2019 Dechuan Sun et al. This is an open access article distributed under the Creative Commons Attribution License, which permits unrestricted use, distribution, and reproduction in any medium, provided the original work is properly cited.

\begin{abstract}
A rocket engine for space propulsion usually has a nozzle of a large exit area ratio. The nozzle efficiency is greatly affected by the nozzle contour. This paper analysed the effect of the constant capacity ratio in Rao's method through the design process of an apogee engine. The calculation results show that increasing the heat capacity ratio can produce an expansion contour of smaller expansion angle and exit area ratio. A simple modification of Rao's method based on thermally perfect gas assumption was made and verified to be more effective. The expansion contour designed by this method has much thinner expansion section and higher performance. For the space engine, a new extension contour type for the end section of the nozzle is proposed. The extension curve bent outward with increasing expansion angle increases the vacuum specific impulse obviously.
\end{abstract}

\section{Introduction}

A de Laval nozzle of large area ratio is generally used in the rocket engines for space propulsion, such as apogee rocket engines that use hypergolic bipropellant combinations. The vacuum specific impulse $\left(I_{\mathrm{sv}}\right)$ of apogee engines is usually above 300 seconds. For example, S400-15 has the specific impulse of 321 seconds at the nominal point [1], R-4D-14 (HPLAE) has demonstrated a specific impulse of $322.2 \pm 2$ seconds [2], and the $I_{\mathrm{sv}}$ of China's second-generation $490 \mathrm{~N}$ apogee engine is 315 seconds $[3,4]$.

For a space engine, to improve the specific impulse is still the goal of engine design because the higher specific impulse means a longer life cycle. To achieve this goal, we can increase the combustion efficiency or nozzle efficiency. However, it is hard to increase the combustion efficiency because higher combustion efficiency means higher combustion temperature, which increases the risk of failure of material or coating system [5-7]. In contrast, increasing the nozzle efficiency is more effective.

The efficiency of a rocket nozzle is affected by many parameters. Manski and Hagemann studied the influence of rocket design parameters on nozzle efficiencies using the nozzle shapes of SSME and Vulcan engines [8]. Their research reveals that increasing chamber pressure leads to an increase of nozzle efficiency and that the kinetic losses raise up to $1 \%$ at lower chamber pressure. Compared with first-stage rocket engines, space engines have lower chamber pressure and smaller size, so their nozzle efficiencies are lower. Arrington et al. tested two small nozzles [9]. Their study shows that the bell nozzle has higher performance than the conical nozzle for the throat Reynolds number range from $2 \times 10^{4}$ to $4 \times 10^{4}$.

For the design of the real rocket nozzle, TIC (Truncated Ideal Contoured), TOC (Thrust Optimized Contoured), TOP (Thrust Optimized Parabolic), and CTIC (Compressed TIC) methods are most commonly used. As summarized by Östlund and Muhammad-Klingmann, the TOC nozzle has the maximum thrust performance except some low altitude application [10]. Therefore, the most widely used design method of the bell nozzle is still the TOC method developed by Rao [11, 12], called Rao's method. Most of the nozzle contours of the bipropellant liquid rocket engines are designed by this method. Östlund and Bigert [13] and Semenov et al. [14] proposed some configurations based on slight modifications of classical bell-shaped nozzles, using Rao's method 


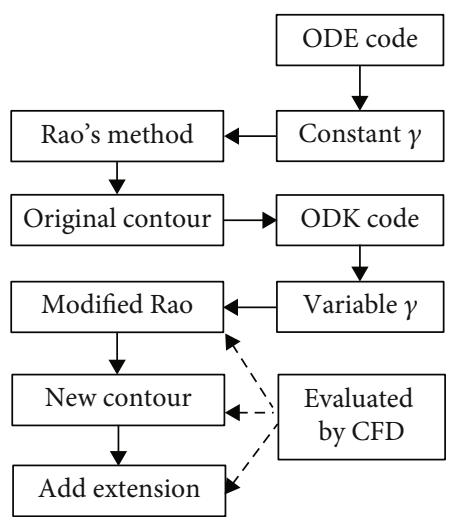

Figure 1: Design procedure. indirectly. Although Rao's method is quite simple, the assumption of inviscid isentropic flow makes it deviate from the real flow. Thus, some direct optimization methods based on computation fluid dynamics (CFD) were studied [15-18]. These methods take into account the viscosity and chemical kinetics in the real flow and show better performance in applications. However, these methods not only are much more complicated than Rao's method but also have little increase on performance of the nozzle of large area ratio. For this reason, we restudied the nozzle design process, hoping that the engine performance can be significantly improved only by the nozzle design.

In this study, taking the $750 \mathrm{~N}$ engine as an example, we made a simple and effective modification on Rao's method. The expansion nozzle contour designed by this method has a slightly higher specific impulse and a smaller exit area ratio. We also proposed a new method to design the end section of the nozzle for the space rocket engine, which can effectively increase the vacuum specific impulse.

\section{Approaches}

2.1. Design Procedure. The design procedure is illustrated in Figure 1. First, an initial nozzle contour is designed by using Rao's method (where $\gamma$, the heat capacity ratio, is calculated by one-dimensional chemical equilibrium code, ODE). Then, its coordinates are inputted into a one-dimensional kinetic code (ODK) to calculate the nonequilibrium flow and the properties of the mixture gas. The specific heat capacity of the real gas is calculated and then substituted into the code of modified Rao's method to redesign a new contour. Finally, a modified nozzle extension section is added to further improve the specific impulse. In the design procedure, the key steps are Rao's method and its modification.

2.2. Equations of Method of Characteristic. Rao's method is based on the method of characteristic (MOC), which is a typical method for calculating the supersonic flow and is discussed in detail by Arrington et al. [9]. MOC uses compatibility equations for the rotational axisymmetric flow:

$$
\rho V d V+d p=0
$$

$$
\begin{aligned}
d p+c^{2} d \rho & =0, \\
\frac{\left(\sqrt{M^{2}-1}\right) d p}{\rho V^{2}} \pm d \theta+\left[\frac{\sin \theta}{y M \cos (\theta \pm \alpha)}\right] & =0 .
\end{aligned}
$$

Compatibility Equations (1) and (2) are held along the streamline, with the characteristic equation:

$$
\frac{d y}{d x}=\tan \theta,
$$

while Equation (3) holds along the left- and right-running Mach lines given by

$$
\frac{d y}{d x}=\tan (\theta \pm \alpha)
$$

2.3. Procedure of Rao's Method. The nozzle design procedure based on Rao's method is described in Reference [9]. As shown in Figure 2, the initial-value line is established by Kliegel's method because it is in reasonable agreement with the measurements of Back et al.; then, the kernel region for the flow field is determined by solving the characteristic line originating from the initial-value line and the wall of throat downstream defined by downstream wall radius $\left(R_{\mathrm{d}}\right)$ and expansion half angle $(\beta)$. After that, the last characteristic line, $B D$, is obtained. To determine the points on the expansion contour, the conservation of mass flow is used; that is, the mass flow through $B D_{1}$ is equal to the mass flow through $D_{1} E_{1}$. When the discrete points $D_{1}, D_{2}$, $D_{3}$, etc. are sequentially selected on $B D$, the points $E_{1}, E_{2}$, $E_{3}$, etc. on the contour can be determined. Finally, according to the criteria of the exit point, the whole expansion contour can be obtained.

2.4. Modification of Rao's Method. In Rao's method, the heat capacity ratio $(\gamma)$ is constant, and the thermodynamic parameters (temperature, pressure, density, and sonic velocity) are all corresponding to the magnitude of velocity. Equations (6a), (6b), (6c), and (6d) give the order of solving these parameters:

$$
\begin{aligned}
T & =T_{\mathrm{s}}-\frac{V^{2}}{2 c_{p}}, \\
c & =\sqrt{\gamma R T}, \\
M & =\frac{|V|}{c}, \\
p & =p_{\mathrm{s}}\left(\frac{T}{T_{\mathrm{s}}}\right)^{\gamma /(\gamma-1)}, \\
\rho & =\frac{p}{R T} .
\end{aligned}
$$




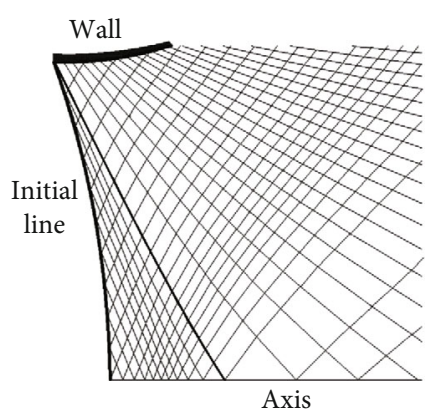

(a)

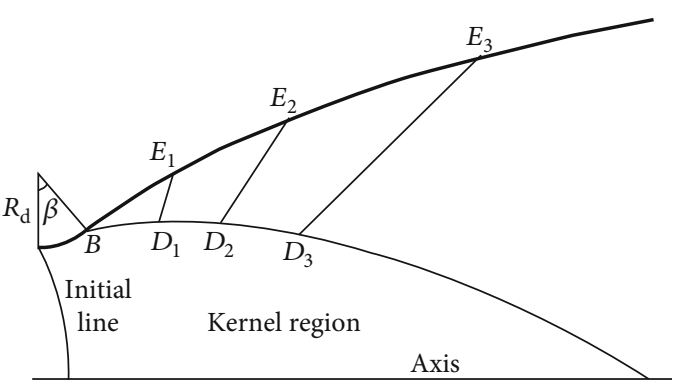

(b)

FIGURE 2: Design procedure of Rao's method: (a) determining the kernel region; (b) determining contour points.

However, for the thermally perfect gas assumption, we solve the temperature through a state equation, and the solving order of parameters is modified as follows:

$$
\begin{aligned}
T & =\frac{p}{\rho R}, \\
c & =\sqrt{\gamma R T}, \\
M & =\frac{|V|}{c},
\end{aligned}
$$

where the heat capacity ratio is calculated by

$$
\gamma=\frac{c_{p}}{c_{p}-R}
$$

In Equation (8), $c_{p}$ is a fitting function of temperature through calculations of ODK. When calling the above subroutine, pressure and density should be predetermined.

2.5. Numerical Simulation Method. We used Fluent, a commercial CFD software, to solve the two-dimensional axisymmetric Navier-Stokes equations to simulate the flow field of the nozzle. An implicit second-order Roe-FDS scheme was used to solve the steady flow equations. Turbulent flow was modelled by using the nonlinear renormalization group (RNG) model, and the enhanced wall function was introduced to treat the near-wall region flow.

\section{Preparation for Design}

In this paper, we used an apogee engine of $750 \mathrm{~N}$ thrust to describe the design process. The engine uses NTO/MMH combination, and the total mixture ratio is 1.65 , which are identical with those of China's $490 \mathrm{~N}$ engines and S400 series of Airbus Space systems. Figure 3 shows the initial contour of the nozzle, which was designed by Rao's method. The chamber pressure $p_{c}$ is $0.85 \mathrm{MPa}$, and the test vacuum specific impulse $I_{\mathrm{sv}}$ is $320.4 \mathrm{~s}$. The nondimensional upstream and downstream wall radii of the nozzle $\left(R_{\mathrm{u}} / R_{\mathrm{t}}\right.$ and $\left.R_{\mathrm{d}} / R_{\mathrm{t}}\right)$ are 1.633 and 0.816 . The expansion half angle $\beta$ is $36.5^{\circ}$.

3.1. Chemical Equilibrium and Kinetic Calculation. For nozzle design and its performance evaluation, chemical equilib-

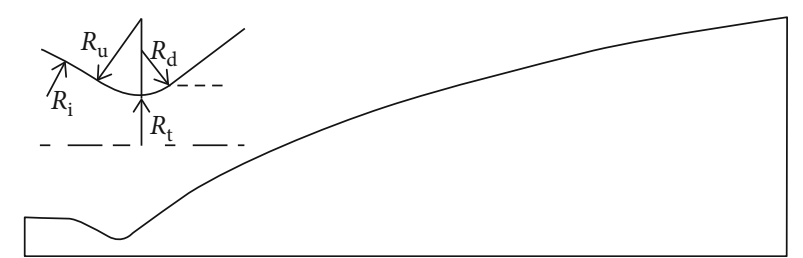

Figure 3: Structure of the nozzle with the area ratio of $210: 1$. It is an $80 \%$ bell-shape nozzle (compared with standard conical nozzle): $R_{\mathrm{t}}=12.25 \mathrm{~mm}, R_{\mathrm{u}} / R_{\mathrm{t}}=1.633, R_{\mathrm{d}} / R_{\mathrm{t}}=0.816$, and $\beta=36.5^{\circ}$.



Figure 4: Vacuum specific impulses at different area ratios. To increase the $I_{\mathrm{sv}}$ (area ratio $=210: 1$ ) by $3 \mathrm{~s}$, the area ratio should be increased to $325: 1$ at least.

rium and kinetic calculation are the key procedures. These calculations can provide all of the data needed in the design and evaluation process. We used an in-house code to calculate the nozzle's theoretical performance and the properties of gas [19]. A C-H-N-O reaction mechanism was used in the calculation because of the use of NTO/MMH combination. The element reactions and the corresponding coefficients of the reaction rate can be found in References [20,21].

Figure 4 shows the theoretical $I_{\mathrm{sv}}$ at different exit area ratios under the chamber pressure of $0.85 \mathrm{MPa}$. For the nozzle with the exit area ratio of $210: 1$, its corresponding theoretical $I_{\mathrm{sv}}$ is about $347.6 \mathrm{~s}$, while the test value is $320.4 \mathrm{~s}$; thus, the total efficiency is about $320.4 / 347.6=92.2 \%$. We 




FIGURE 5: Comparison of the heat capacity ratio obtained with different assumptions.

TABLE 1: Calculation results with different grid numbers.

\begin{tabular}{lccc}
\hline Grid number & $F_{\mathrm{v}}(\mathrm{N})$ & $F_{\mathrm{m}}(\mathrm{N})$ & $F_{\mathrm{p}}(\mathrm{N})$ \\
\hline $288 \times 60$ & 735.897 & 715.096 & 20.801 \\
$288 \times 80$ & 735.895 & 715.092 & 20.803 \\
$288 \times 100$ & 735.901 & 715.108 & 20.793 \\
$288 \times 120$ & 735.926 & 715.133 & 20.793 \\
\hline
\end{tabular}

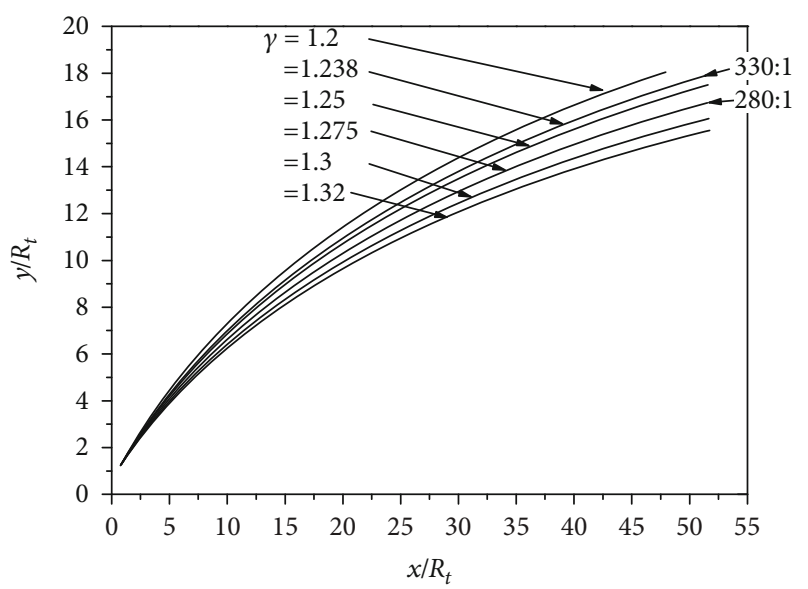

FIGURE 6: Expansion contours designed by various constant $\gamma$ values.

can see that in order to increase the specific impulse, the exit area ratio must be increased.

Figure 5 shows the variation of heat capacity ratio $\gamma$ at different nozzle area ratios. $\gamma$ obtained by using the chemical equilibrium assumption is quite different from that obtained by using the frozen or kinetic flow assumption and presents a smaller value and tends to be constant. The reason is that the composition of the gas changes instantaneously with the temperature under the equilibrium assumption. However, it is impossible in the real nozzle flow. The smaller the nozzle size is, the slower the change of the gas composition is than the flow, and the closer the gas property is to the frozen flow. Thus, the result of kinetics is closest to the real state. Because the calculation of chemical kinetics is related to the size of the nozzle, we used the initial nozzle contour to calculate the chemical reaction flow in the nozzle under the assumption that the nozzle contour has little change and fitted the relationship between the specific heat at constant pressure $\left(c_{p}\right)$ and temperature:

$$
c_{p}=a_{0}+a_{1} T+a_{2} T^{2}+a_{3} T^{3}+a_{4} T^{4} .
$$

It is a fitting polynomial. For $T \geq 1000 \mathrm{~K}, a_{0}=$ $1188.27296, a_{1}=0.74502, a_{2}=-2.15067 E-4, a_{3}=2.61593 E$ 8 , and $a_{4}=-7.06741 E-13$, and for $T<1000 \mathrm{~K}, a_{0}=$ $1476.10931, a_{1}=-0.14838, a_{2}=7.94836 E-4, a_{3}=-4.60444 E$ -7 , and $a_{4}=8.11403 E-11$. It should be noted that although the expression of this $c_{p}$ is identical with that of JANNAF database [21], the composition of the gas varies. In modification of Rao's method, this expression is substituted into Equation (8) to solve $\gamma$.

Viscosity of the gas was also treated as a function of temperature:

$$
\begin{aligned}
\mu= & 5.65752 \times 10^{-6}+3.71243 \times 10^{-8} \mathrm{~T} \\
& -5.00581 \times 10^{-12} T^{2}+4.3062 \times 10^{-16} T^{3} .
\end{aligned}
$$

3.2. Verification of Numerical Simulation. To verify the accuracy of the numerical simulation method and grid independence, we calculated the flow field of the initial nozzle with different numbers of the grid and then compared them with the test. Table 1 lists the calculation results. We can see that the vacuum thrusts $F_{\mathrm{v}}$ calculated by different grids are almost equal. This means that these grids can meet the requirement of calculation. Thus, we selected the grid of $288 \times 80$ to simulate all the cases.

By integrating the parameters at the nozzle exit, we can obtain axial momentum thrust $F_{\mathrm{m}}=715.092 \mathrm{~N}$ and pressure thrust $F_{\mathrm{p}}=20.803 \mathrm{~N}$. Thus, the total vacuum thrust $F_{\mathrm{v}}=F_{\mathrm{m}}+F_{\mathrm{v}}=735.895 \mathrm{~N}$, and the vacuum specific impulse $\quad I_{\mathrm{sv}}=F_{\mathrm{v}} / \dot{m}=735.895 / 0.235=3131.468 \mathrm{~N} \cdot \mathrm{s} / \mathrm{kg}=$ $319.3 \mathrm{~s}$. This is very close to the average value of hot fire tests, $320.4 \mathrm{~s}$, and the deviation is $-0.34 \%$. Therefore, the numerical simulation results can be used to evaluate the nozzle design.

\section{Results and Discussion}

As descripted in Figure 4, higher performance needs a higher area ratio; the aim area ratio is $330: 1$. In our study, the length of the expansion section was limited to be $80 \%$ length of a 15-degree conical nozzle with the area ratio of $330: 1, x_{e} / R_{\mathrm{t}} \approx 51.5$.

4.1. Influence of Constant $\gamma$. First, we used various constant $\gamma$ values in Rao's method to design the expansion contours, which are shown in Figure 6. With the increase of $\gamma$ value, the expansion half angle $\beta$ and the exit area ratio of the contour become smaller. 
TABle 2: Performance of the nozzle with various contours.

\begin{tabular}{lcccc}
\hline$\gamma$ & $I_{\mathrm{sv}}(\mathrm{s})$ & $F_{\mathrm{v}}(\mathrm{N})$ & $F_{\mathrm{m}}(\mathrm{N})$ & $F_{\mathrm{p}}(\mathrm{N})$ \\
\hline 1.2 & 320.19 & 737.90 & 718.29 & 19.61 \\
1.238 & 321.03 & 739.83 & 720.49 & 19.39 \\
1.25 & 321.06 & 739.83 & 720.43 & 19.41 \\
1.275 & 321.08 & 739.89 & 720.34 & 19.55 \\
1.3 & 321.05 & 739.80 & 720.11 & 19.69 \\
1.32 & 320.93 & 739.59 & 719.76 & 19.84 \\
\hline
\end{tabular}

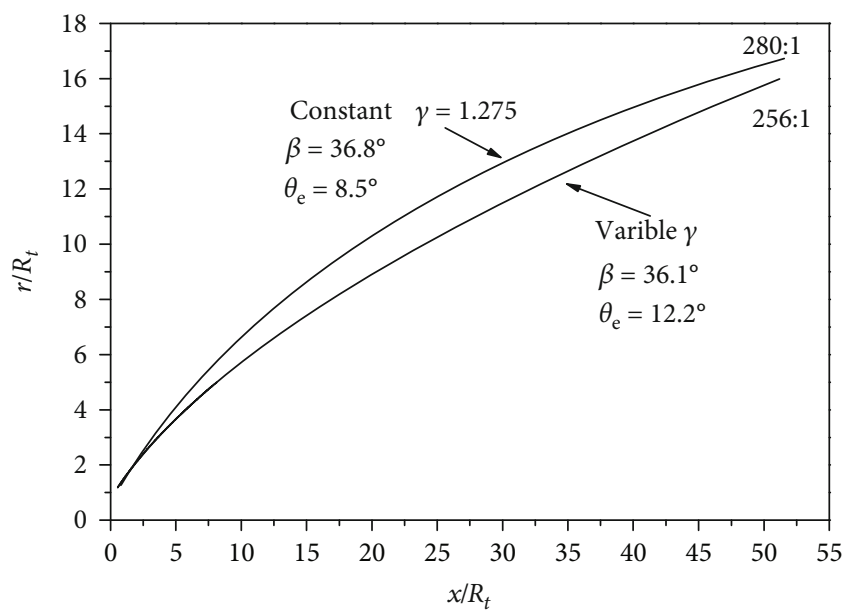

FIgURE 7: Comparison of contours designed with constant and variable $\gamma$.

Table 2 lists the calculation results of the nozzle flow filed with the contours in Figure 6. The contour designed with $\gamma=1.2$ shows the smallest $I_{\mathrm{sv}}$ and $F_{\mathrm{v}}$. For larger $\gamma$ from 1.238 to 1.3 , different contours show nearly the same $I_{\text {sv }}$ and $F_{\mathrm{v}}$, and the one designed with $\gamma=1.275$ shows the maximum performance. With the increase of $\gamma$, the contour becomes thinner. The calculation results also show that Rao's method with constant $\gamma$ can only increase $I_{\text {sv }}$ by $1.78 \mathrm{~s}$ (321.08-319.3); this value is less than the theoretical prediction.

4.2. Effect of $\gamma$. As described in Section 3.1, heat capacity of the gas changes rapidly due to the change of temperature near the nozzle throat. Thus, using $\gamma$ calculated by Equation (8) is more reasonable than using constant $\gamma$.

Figure 7 shows the comparison of the contours designed by Rao's method with constant $\gamma$ and modified Rao's method with variable $\gamma$. The new contour designed with variable $\gamma$ has a smaller expansion angle $(\beta)$, larger exit angle $\left(\theta_{\mathrm{e}}\right)$, and smaller exit area ratio as shown in Figure 7.

Table 3 lists the CFD results of these contours, where "Var." represents variable $\gamma$ and "Var.+dis." represents the contour designed with variable $\gamma$ and boundary layer displacement. We can see that $I_{\text {sv }}$ can increase one second more than the nozzle contour with the area ratio of $280: 1$ and $2.7 \mathrm{~s}$ more than the original contour with the area ratio of $210: 1$, by using modified Rao's method.

Figure 8 compares the distribution of the Mach number in different nozzles designed by variable and constant $\gamma$.
TABle 3: Performance of the nozzle designed by different methods.

\begin{tabular}{lcccc}
\hline$\gamma$ & $I_{\mathrm{sv}}(\mathrm{s})$ & $F_{\mathrm{v}}(\mathrm{N})$ & $F_{\mathrm{m}}(\mathrm{N})$ & $F_{\mathrm{p}}(\mathrm{N})$ \\
\hline 1.275 & 321.08 & 739.89 & 720.34 & 19.55 \\
Var. & 322.00 & 742.07 & 723.57 & 18.50 \\
Var.+dis. & 322.09 & 742.28 & 724.08 & 18.20 \\
\hline
\end{tabular}
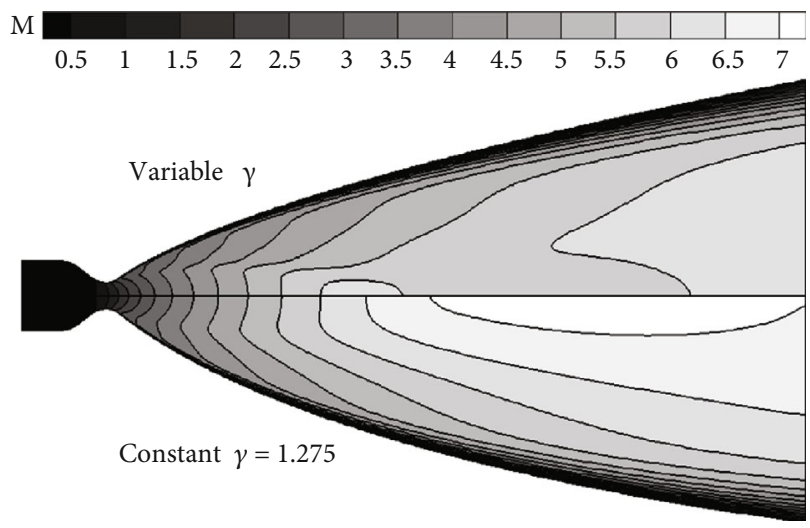

Figure 8: Comparison of Mach numbers in different nozzles.

We can see that the Mach number in the nozzle designed by variable $\gamma$ is more uniform although it is smaller than that of constant $\gamma$ near the exit zone. Figure 9 compares the pressure and axial velocity at the nozzle exit. We can observe that the axial velocity at the exit is relatively uniform and almost the same, but the pressure at the exit of the nozzle by variable $\gamma$ is more uniform, thus generating higher pressure thrust.

4.3. The Off-Design Performance. For space propulsion, rocket engines generally work on nominal condition. However, they work on off-design conditions sometimes, and the mass flow rates are greater than or less than the nominal value. Thus, we compared the performance of the nozzles designed by Rao's method and modified Rao's method. Table 4 shows the calculation results. We can observe that $I_{\text {sv }}$ increases slightly with the increase of the mass flow rate. For all off-design conditions, the nozzle designed by modified Rao's method presents higher performance, and the highest increase of $I_{\mathrm{sv}}$ is on the design point.

4.4. Extension of the Nozzle. In this section, we studied how to achieve an obvious increase of $I_{\text {sv }}$ because the above effort can only increase $I_{\mathrm{sv}}$ by $2.7 \mathrm{~s}$. A longer nozzle is the only selection. However, since the expansion contour has a smaller expansion angle at the exit of the nozzle, the convex or straight extension curve can bring a smaller area ratio increment and lead to additional viscous losses. Therefore, the increase of $I_{\mathrm{sv}}$ caused by the convex extension curve is not large. We proposed a new idea that a smooth curve with an increasing expansion angle will have a larger benefit. Figure 10 shows four different extension curves studied in this paper, marked as L1, L2, L3, and L4. L1 is straight, and the others curve outward. All these extension contours were connected smoothly with the $80 \%$ length nozzle designed by modified Rao's method. 


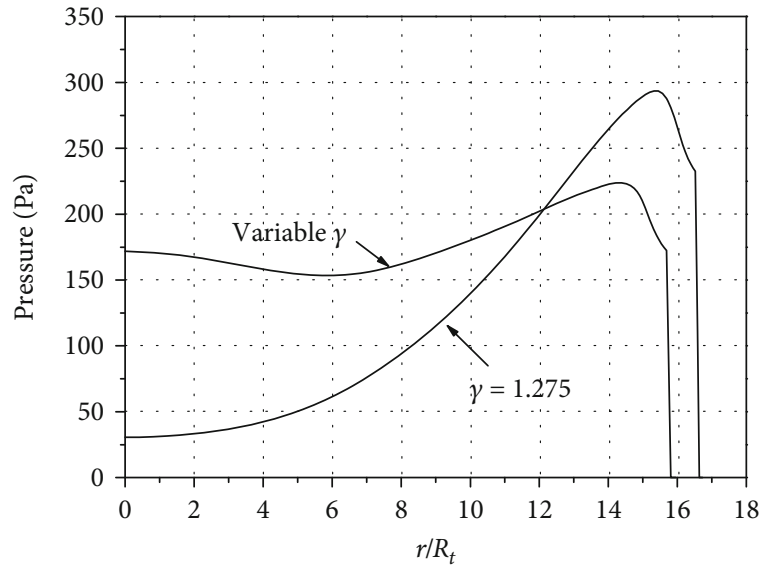

(a) Pressure

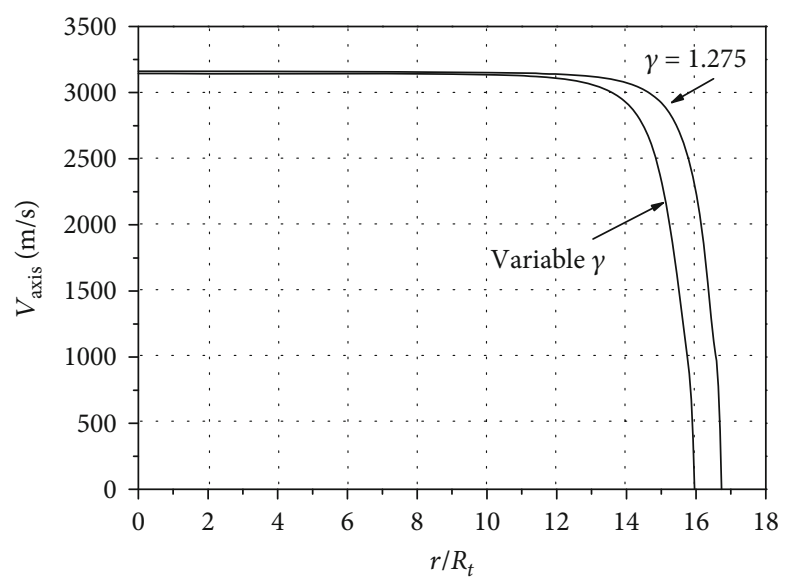

(b) Axial velocity

FIGURE 9: Comparison of Mach numbers in different nozzles.

TABle 4: Performance of the nozzle designed by different methods.

\begin{tabular}{lcc}
\hline \multirow{2}{*}{ Mass flow } & $\gamma=1.275$ & Var. $\gamma$ \\
& $F_{\mathrm{v}}(\mathrm{N}) / I_{\mathrm{sv}}(\mathrm{s})$ & $F_{\mathrm{v}}(\mathrm{N}) / I_{\mathrm{sv}}(\mathrm{s})$ \\
\hline$-20 \%$ & $590.98 / 320.55$ & $592.22 / 321.22$ \\
$-10 \%$ & $665.73 / 320.97$ & $667.14 / 321.65$ \\
Nominal & $739.89 / 321.08$ & $742.07 / 322.00$ \\
$+10 \%$ & $813.99 / 321.10$ & $815.85 / 321.83$ \\
$+20 \%$ & $888.78 / 321.38$ & $890.58 / 322.04$ \\
\hline
\end{tabular}

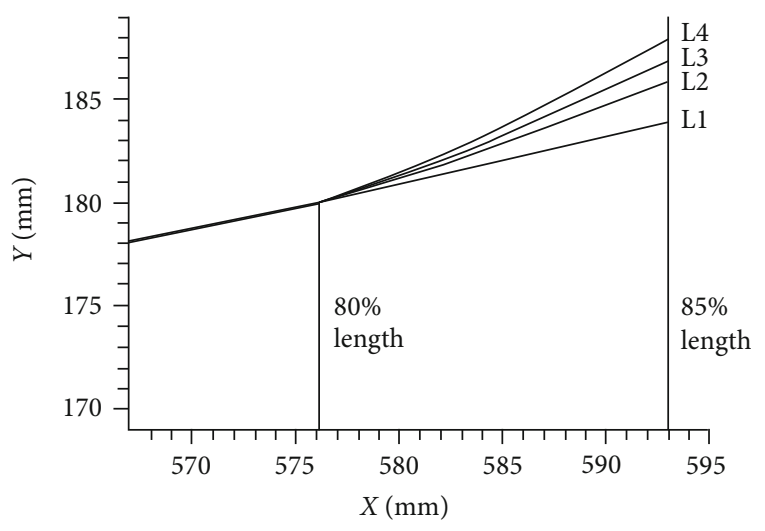

FIGURE 10: Comparison of various extension curves.

Table 5 lists the CFD results of these contours. Compared with the maximum thrust contour with the area ratio of $256: 1$, the straight extension line (L1) shows an increase of $I_{\mathrm{sv}}$ by only $0.42 \mathrm{~s}$. Moreover, the other extension curves have an evident increase of $I_{\mathrm{sv}}$. Among all the curves, L3 has the highest $I_{\text {sv }}$. By comparing each column data in Table 5, we can observe that the increase of thrust mainly depends on the increase of momentum thrust $F_{\mathrm{m}}$ and the variation of pressure thrust $F_{\mathrm{p}}$, and viscous drag of the wall $D_{w}$ changes little.

This phenomenon can be explained by the PrandtlMeyer flow downstream of point $E$. The expansion wave (in the case of L2, L3, and L4) originating from the curve accelerates the main flow to increase $F_{\mathrm{m}}$ and $F_{r}$. If the expansion
TABle 5: Performance of the nozzle with various extension contours.

\begin{tabular}{lccccc}
\hline & $I_{\mathrm{sv}}(\mathrm{s})$ & $F_{\mathrm{v}}(\mathrm{N})$ & $F_{\mathrm{m}}(\mathrm{N})$ & $F_{\mathrm{p}}(\mathrm{N})$ & $D_{\mathrm{w}}(\mathrm{N})$ \\
\hline Var.+dis. & 322.09 & 742.28 & 724.08 & 18.20 & 36.97 \\
L1 & 322.42 & 743.03 & 724.69 & 18.34 & 37.65 \\
L2 & 323.32 & 745.10 & 726.79 & 18.31 & 37.68 \\
L3 & 323.71 & 746.02 & 727.85 & 18.18 & 37.70 \\
L4 & 323.64 & 745.84 & 727.67 & 18.16 & 37.71 \\
\hline
\end{tabular}

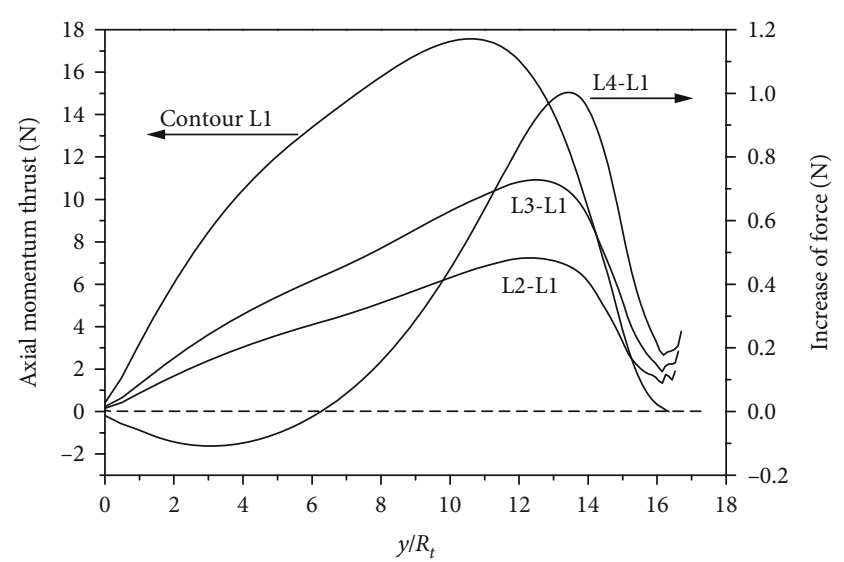

Figure 11: Axial momentum force along the radius.

angle is too large, the axial thrust will decrease (L4). Figure 11 shows the distribution of axial momentum force $\left(\rho V_{x}\right)$ for L1 and the differences between other extension curves and L1, marked as L2-L1, L3-L1, and L4-L1. It can be observed that the axial momentum force at the exit increases with the extension curve moving outward (L2-L1 and L3-L1) and then decreases in the core region (L4).

\section{Conclusions}

This paper made a modification of Rao's method and proposed a new idea on design of the nozzle extension. Through 
a concrete design process of the nozzle of an apogee engine, the methods were verified to be more effective than the traditional method. Based on the calculation results, we draw some conclusions.

The heat capacity ratio used in Rao's method plays a great role in design. For a given length of nozzle, the nozzle contour designed with a larger specific heat ratio has a significantly smaller area ratio, and the nozzle performance is almost identical, which is very beneficial to reduce the nozzle weight.

The expansion contour designed by using the variable heat capacity ratio or thermally perfect gas assumption has a much thinner expansion section than that designed by using the constant capacity ratio or caloric perfect gas assumption. Compared with the original design, the exit area ratio of the nozzle designed by this method not only reduces significantly from $330: 1$ to $256: 1$ but also has an increase of the vacuum specific impulse by about 1 second at the design point. The calculation results of off-design performance also show the effectiveness of the method. Furthermore, this method is simple and easy to implement.

For the space rocket engine, we proposed an innovative nozzle extension section, which is an extension curve bent outward with the increasing expansion angle. Mounting such a section at the end of the nozzle will significantly increase the vacuum specific impulse, resulting in an additional gain of $1.5 \mathrm{~s}$ or even higher.

\section{Nomenclature}

c: $\quad$ Speed of sound

$c_{p}$ : Specific heat capacity at constant pressure

$D_{\mathrm{w}}$ : Viscous drag of nozzle wall

$F_{\mathrm{m}}$ : Momentum thrust

$F_{\mathrm{p}}$ : Pressure thrust

$F_{\mathrm{v}}$ : Vacuum thrust

$I_{\mathrm{sv}}$ : Vacuum specific impulse

M: Mach number

$p: \quad$ Static pressure

$p_{c}$ : Combustion chamber pressure

$p_{s}$ : Stagnation pressure

$R: \quad$ Gas constant

$R_{\mathrm{d}}$ : Downstream wall radius

$R_{\mathrm{i}}$ : Inlet wall radius

$R_{\mathrm{t}}$ : Throat radius

$R_{\mathrm{u}}$ : Upstream wall radius

T: Temperature

$T_{\mathrm{s}}$ : Stagnation temperature

$V$ : Velocity

$\alpha$ : Mach angle

$\beta$ : Expansion half angle

$\gamma$ : Heat capacity ratio

$\rho:$ Density

$\theta$ : $\quad$ Flow angle.

\section{Data Availability}

The data used to support the findings of this study are included within the article.

\section{Conflicts of Interest}

The authors declare that there is no conflict of interest regarding the publication of this paper.

\section{Acknowledgments}

We would like to thank the Shanghai Institute of Space Propulsion for providing the test data employed in this study.

\section{References}

[1] http://www.space-propulsion.com/spacecraft-propulsion/ apogee-motors/index.html.

[2] C. Stechman, P. Woll, R. Fuller, and A. Colette, "A high performance liquid rocket engine for satellite main propulsion," in 36th AIAA/ASME/SAE/ASEE Joint Propulsion Conference and Exhibit, Las Vegas, NV, USA, 2000.

[3] C. Liu, J. Chen, H. Han, Y. Wang, and Z. Zhang, "A long duration and high reliability liquid apogee engine for satellites," Acta Astronautica, vol. 55, no. 3-9, pp. 401-408, 2004.

[4] H. Zhou, F. Xu, X. Zhang, and Z. Jia, "Effect of thickness on oxidation resistance of coatings on $490 \mathrm{~N}$ apogee engine," Aerospace Materials \& Technology, vol. 41, no. 5, pp. 66-68, 2011.

[5] T. Fiedler, M. Bäker, and J. Rösler, "Large heat flux exposure of metallic coatings for rocket engine applications," Surface and Coatings Technology, vol. 332, no. 25, pp. 30-39, 2017.

[6] X. Zhang, "Coupled simulation of heat transfer and temperature of the composite rocket nozzle wall," Aerospace Science and Technology, vol. 15, no. 5, pp. 402-408, 2011.

[7] D. Sciti, L. Zoli, L. Silvestroni, A. Cecere, G. D. di Martino, and R. Savino, "Design, fabrication and high velocity oxy-fuel torch tests of a $\mathrm{C}_{\mathrm{f}}-\mathrm{ZrB}_{2}$ - fiber nozzle to evaluate its potential in rocket motors," Materials \& Design, vol. 109, pp. 709-717, 2016.

[8] D. Manski and G. Hagemann, "Influence of rocket design parameters on engine nozzle efficiencies," Journal of Propulsion and Power, vol. 12, no. 1, pp. 41-47, 1996.

[9] L. A. Arrington, B. D. Reed, and A. Rivera Jr., "A Performance comparison of two small rocket nozzles," in 32nd Joint Propulsion Conference and Exhibit, Lake Buena Vista, Fl, USA, July 1996.

[10] J. Östlund and B. Muhammad-Klingmann, "Supersonic flow separation with application to rocket engine nozzles," Applied Mechanics Reviews, vol. 58, no. 3, pp. 143-177, 2005.

[11] G. V. R. Rao, "Exhaust nozzle contour for optimum thrust," Journal of Jet Propulsion, vol. 28, no. 6, pp. 377-382, 1958.

[12] M. J. Zucrow and J. D. Hoffman, Gas Dynamics, Wiley, New York, NY, USA, 1976.

[13] J. Östlund and M. Bigert, "A sub scale investigation on side loads in sea level rocket nozzles," in 35th Joint Propulsion Conference and Exhibit, Los Angeles, CA, USA, 1999.

[14] V. V. Semenov, S. L. Finogenov, I. E. Ivanov, I. A. Krykov, and A. A. Talalayev, "Liquid propulsion with altitude compensation concept trade study," in 57th International Astronautical Congress, Valencia, Spain, October 2006.

[15] M. Yumusak and S. Eyi, "Design optimization of rocket nozzles in chemically reacting flows," Computer \& Fluids, vol. 65, pp. 25-34, 2012. 
[16] A. A. Ezertas, M. Yumusak, and S. Eyi, "Design optimization of rocket nozzles," in 46th AIAA/ASME/SAE/ASEE Joint Propulsion Conference \& Exhibit, Nashville, TN, USA, July 2010.

[17] H. Ogawa and R. R. Boyce, "Nozzle design optimization for axisymmetric scramjets by using surrogate-assisted evolutionary algorithms," Journal of Propulsion and Power, vol. 28, no. 6, pp. 1324-1338, 2012.

[18] K. Tanimizu, D. J. Mee, R. J. Stalker, and P. A. Jacobs, “Thrust nozzle design study for a quasi-axisymmetric scramjetpowered vehicle," Journal of Propulsion and Power, vol. 27, no. 1, pp. 40-49, 2011.

[19] D. Sun, J. Chen, and Q. Lin, "Chemical kinetics analysis for nozzle performance," Journal of Propulsion Technology (China), vol. 24, no. 3, pp. 222-224, 2003.

[20] D. Sun and Z. Yu, "A nozzle contour optimization method considering the change in fuel gas properties," Acta Armamentarii, vol. 39, no. 11, pp. 2145-2152, 2018.

[21] G. R. Nickerson, D. E. Coats, and J. L. Bartz, "The twodimensional kinetic (TDK) reference computer program: engineering and programing manual," 1973, NASA-CR-152999. 


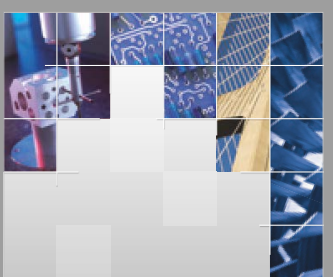

\section{Enfincering}
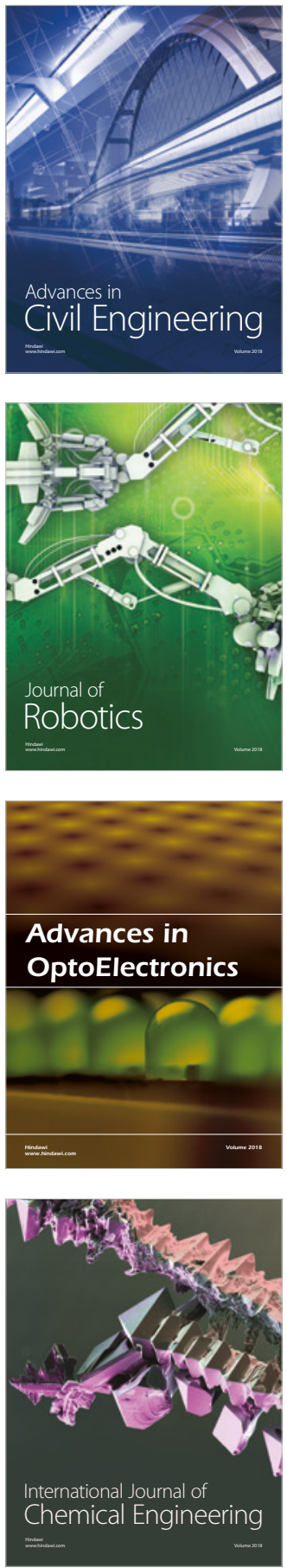

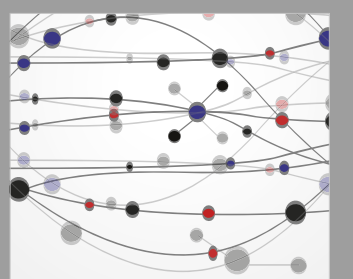

\section{Rotating \\ Machinery}

The Scientific World Journal

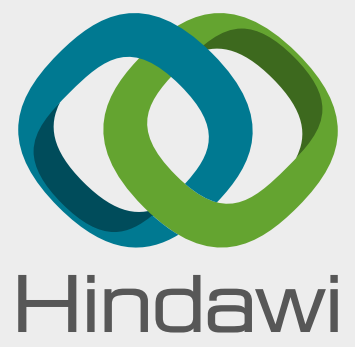

Submit your manuscripts at

www.hindawi.com
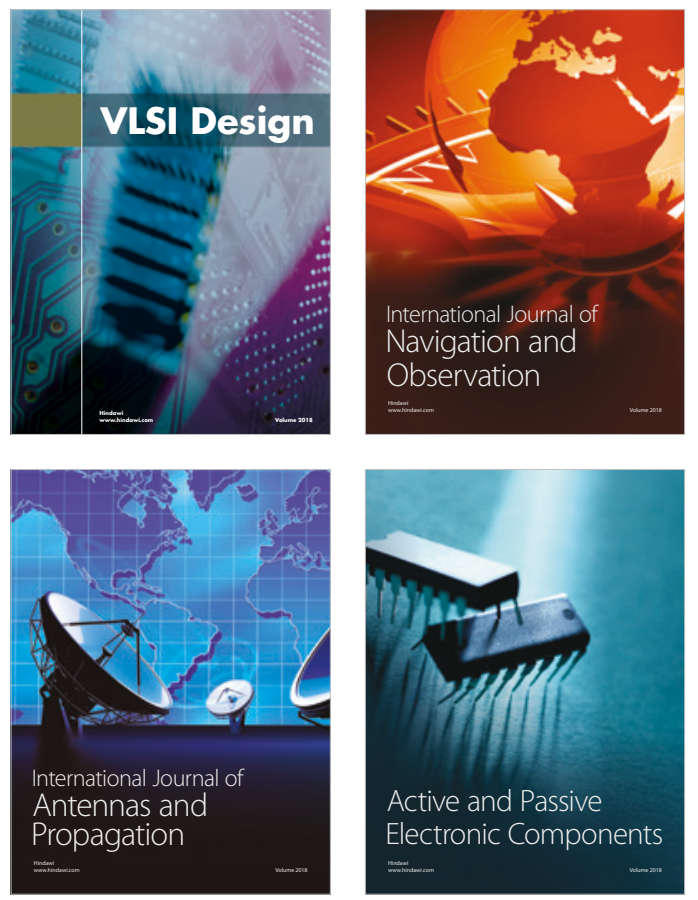
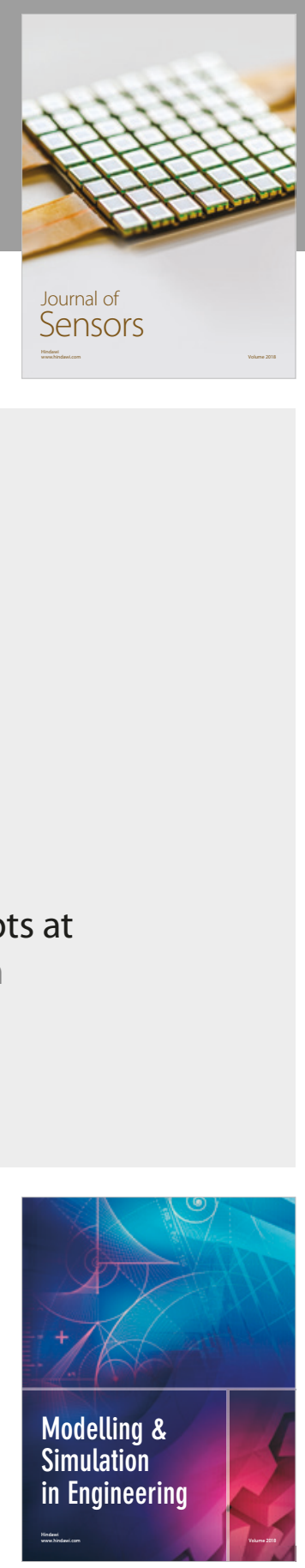

\section{Advances \\ Multimedia}
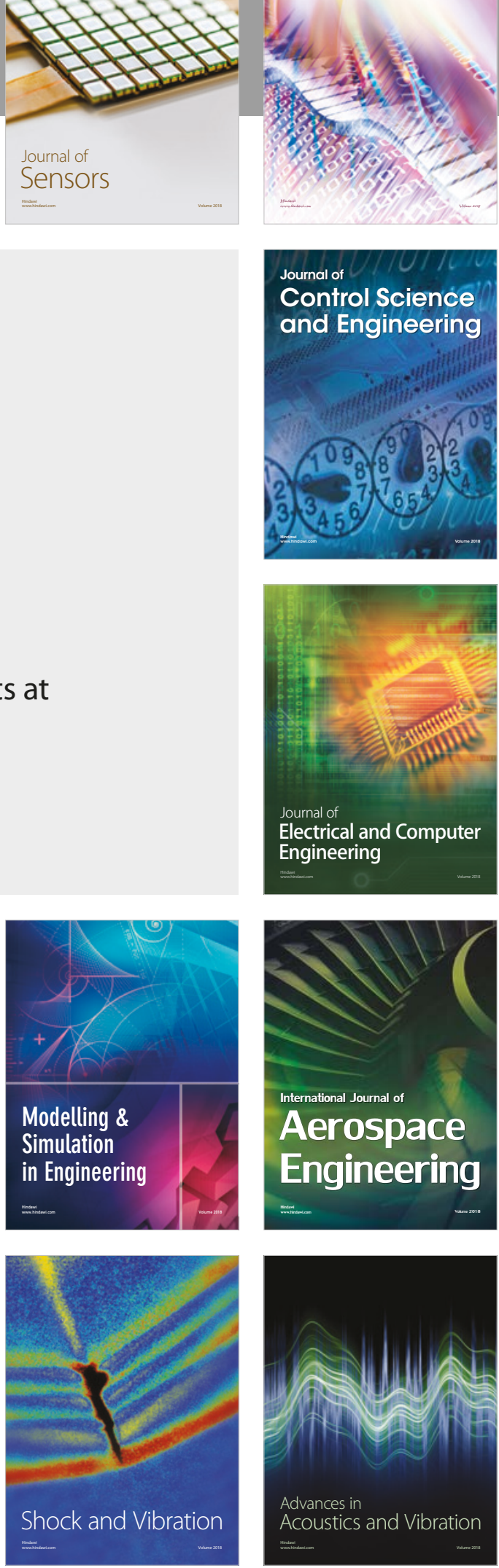